VOL. $3(1970), 185-192$.

\title{
A remark on primes in arithmetic progressions
}

\section{Ronald Alter and Mark Villarino}

\begin{abstract}
By generalizing a technique of Landau, the authors prove that the excess of the number of primes of the form $10 x \pm 3$ over the number of primes of the form $10 x \pm 1$ is infinite.
\end{abstract}

\section{Introduction}

In 1853, Chebyshev conjectured the still unproven assertion that there exist more primes of the form $4 y+3$ than of the form $4 y+1$ in the sense that

$$
\lim _{x \rightarrow+\infty} \sum_{p>2}(-1)^{\frac{p-1}{2}} e^{-\frac{p}{x}}=-\infty .
$$

Knapowski and Turán's [3] investigations into this conjecture point out that there are really four classes of problems, there labeled (a) - (d), involved. They also state that their investigations deal with classes (c) and (d) only.

This paper concerns itself with a problem from class (a). We begin with a weaker form of Chebyshev's conjecture. If

$$
\begin{gathered}
\Pi(x, k, z)=\sum_{\substack{p \leq x \\
p \equiv Z(k)}} 1 \\
P\left(x, k, z_{1}, z_{2}\right)=\Pi\left(x, k, z_{1}\right)-\Pi\left(x, k, z_{2}\right),
\end{gathered}
$$

then 


$$
P(x, 4,3,1) \rightarrow \infty \text { as } x \rightarrow \infty .
$$

Landau [4] proves Chebyshev's theorem. He also points out that Phragmén [6] had given the only previous correct, but very complicated, proof.

In this paper, by generalizing Landau's technique, the authors prove that

$$
P(x) \equiv P(x, 10, \pm 3, \pm 1) \rightarrow \infty \text { as } x \rightarrow \infty \text {. }
$$

More precisely, it is proven that there exists an infinite sequence $\left\{x_{n}\right\}$ such that

$$
x_{1}<x_{2}<\ldots+\infty
$$

and that as $n \rightarrow \infty$

$$
\frac{\pi\left(x_{n}, 10, \pm 3\right)-\Pi\left(x_{n}, 10, \pm 1\right)}{\left(\frac{\sqrt{x_{n}}}{\log x_{n}}\right)} \rightarrow 1 .
$$

\section{Main results}

The following theorem on Dirichlet series is well known.

THEOREM 1 (Landau). Suppose the series $\sum_{n=1}^{\infty} \frac{a_{n}}{n^{s}}$ converges for $s>\alpha$ and that $a_{n} \geq 0$ for $n \geq n_{0}$. Choose $\beta<\alpha$ and suppose its sum function, $f(s)$, is regular for $\alpha \geq s>\beta$ when continued along the real axis. Then the series converges for $s>B$.

In the next two theorems the desired result (3) is achieved. In order to establish them the following is needed.

LEMMA 1. For all complex $s=\sigma+i t$ it follows that

$$
\left|\frac{1}{n^{s}}-\frac{1}{(n+1)^{s}}-\frac{s}{n^{s+1}}\right| \leq \frac{|s||s+1|}{n^{\sigma+2}} \text {. }
$$

Proof. For $s=0$ inequality $(4)$ is immediate. For $s \neq 0$ it follows from 


$$
\begin{aligned}
\frac{1}{n^{s}}-\frac{1}{(n+1)^{s}}-\frac{s}{n^{s+1}} & =s \int_{n}^{n+1}\left(\frac{1}{u^{s+1}}-\frac{1}{n^{s+1}}\right) d u \\
& =s(s+1) \int_{n}^{n+1} d u \int_{u}^{n} \frac{d v}{v^{s+2}} .
\end{aligned}
$$

LEMMA 2. For $y \geq 0$ and $\frac{1}{2} \leq s \leq 1$ it follows that

$$
(10 y+1)^{-8}-(10 y+3)^{-5}-(10 y+7)^{-5}+(10 y+9)^{-s}>0 .
$$

Proof. Recall the "power" inequalities, [2], that for $\alpha, \beta$ real, $0<\beta<1<\alpha$ and $0<s \neq 1$ we have

$$
\alpha^{s}>1+s(\alpha-1) \text { and } \beta^{s}>1-s(1-\beta) \text {. }
$$

By appropriate substitution and addition it follows that

$$
\frac{1}{1+s(10 y+2)}+\frac{1}{1+s(10 y+6)}>\frac{1}{(10 y+3)^{s}}+\frac{1}{(10 y+7)^{s}}
$$

and

$$
\frac{1}{(10 y+1)^{s}}+\frac{1}{(10 y+9)^{s}}>2-s\left(\frac{10 y}{10 y+1}\right)-s\left(\frac{10 y+8}{10 y+9}\right) \text {. }
$$

Clearly inequality (5) follows once we can establish the stronger

$$
2>s\left(\frac{10 y}{10 y+1}\right)+s\left(\frac{10 y+8}{10 y+9}\right)+\frac{1}{1+s(10 y+2)}+\frac{1}{1+s(10 y+6)} \text {. }
$$

We note that a simple computation shows that (6) holds for $s=1$ and $y>0$. Also, it is easy to establish that the right hand side of inequality $(6)$ is a continuous, strictly increasing function of $s$ in the interval $\left[\frac{1}{2}, 1\right]$. Hence, since (6) holds for $s=1$ it certainly holds for $\frac{1}{2} \leq s \leq 1$ and this proves the lemma.

We define the non-principal character, $\chi(n),(\bmod 10)$ as

$$
\chi(n)=\left\{\begin{array}{cll}
1 & \text { if } n \equiv \pm 1 & (\bmod 10) \\
-1 & \text { if } n \equiv \pm 3 \quad(\bmod 10) \\
0 & \text { otherwise. } &
\end{array}\right.
$$

THEOREM 2. Let $s=\sigma+i t$ and put

$$
F(s)=\sum_{n=2}^{\infty} \frac{P(n)-(\sqrt{n} / \log n)}{n^{6+1}}
$$


so that $F(s)$ is analytic for $\sigma>1$. Then by direct continuation $F(s)$ is regular for $1 \geq s>\frac{1}{2}$. The point $s=\frac{1}{2}$ is a singular point of $F(x)$ and yet $\lim _{s \rightarrow \frac{1}{2}^{+}} F(x)$ exists.

Proof. Using the notation of Landau [5] we let $R_{1}(s), R_{2}(s), \ldots$ denote functions of $s$ which are regular for $\sigma>1$ and which $c$ an be analytically continued along the real segment $\frac{1}{2} \leq s \leq 1$. Recalling the character, $\chi(n)$, we let

$$
\begin{aligned}
L(s)=\sum_{n=1}^{\infty} \frac{x(n)}{n^{s}}=1- & \frac{1}{3^{s}}-\frac{1}{7^{s}}+\frac{1}{9^{s}}+\frac{1}{11^{s}}+\ldots+ \\
& +\frac{1}{(10 y+1)^{s}}-\frac{1}{(10 y+3)^{s}}-\frac{1}{(10 y+7)^{s}}+\frac{1}{(10 y+9)^{s}}+\ldots .
\end{aligned}
$$

It follows, by Lemma 2 , that $L(s)>0$ for $\frac{1}{2} \leq s \leq 1$. Hence, in this interval, we can define

$$
\log L(s)=\sum_{p, m} \frac{x\left(p^{m}\right)}{m p^{s}}=R_{1}(s)
$$

For $\sigma>1$ we have

$$
\sum_{p, m} \frac{\chi\left(p^{m}\right)}{m p^{s}}=\sum_{p} \frac{\chi(p)}{p^{s}}+\frac{1}{2} \sum_{p} \frac{\chi\left(p^{2}\right)}{p^{2 s}}+R_{2}(s) \ldots
$$

Thus,

$$
\sum_{p} \frac{\chi(p)}{p^{s}}=-\frac{1}{2} \sum_{p} \frac{\chi\left(p^{2}\right)}{p^{2 s}}+R_{3}(s)=-\frac{1}{2} \sum_{p} \frac{1}{p^{2 s}}+R_{4}(s) .
$$

On the other hand letting $\zeta(s)$ be the Riemann zeta function, it follows that

$$
\log \left(\left(s-\frac{1}{2}\right) \zeta(2 s)\right)=\log \zeta(2 s)+\log \left(s-\frac{1}{2}\right)=\mathrm{R}_{5}(s)
$$

and

$$
\log \zeta(2 s)=\sum_{p} \frac{1}{p^{2 s}}+R_{6}(s)
$$

This implies that

$$
\sum_{p} \frac{1}{p^{2 s}}=-\log \left(s-\frac{1}{2}\right)+R_{7}(s)
$$


and

$$
\sum_{p} \frac{x(p)}{p^{s}}=\frac{1}{2} \log \left(s-\frac{1}{2}\right)+R_{8}(s) .
$$

This says that the series on the left, in the last two equations, are convergent for $s>\frac{1}{2}$.

Recalling the definitions of $\pi(x, 10, \pm 1)$ and $\pi(x, 10, \pm 3)$. it. follows that

$$
\begin{aligned}
& \Pi(n, 10, \pm 1)-\Pi(n-1,10, \pm 1)=\left\{\begin{array}{l}
1 \text { if } n \text { is a prime } 10 y \pm 1 \\
0 \text { otherwise. }
\end{array}\right. \\
& \Pi(n, 10, \pm 3)-\Pi(n-1,10, \pm 3)= \begin{cases}1 \text { if } n \text { is a prime } 10 y \pm 3 \\
0 \text { otherwise. }\end{cases}
\end{aligned}
$$

Recalling (2) it now follows that

$$
\begin{aligned}
-\sum_{n=2}^{\infty} \frac{P(n)-P(n-1)}{n^{s}} & \\
& =\sum_{n=2}^{\infty} \frac{[\Pi(n, 10, \pm 1)-\Pi(n-1,10, \pm 1)]-[\Pi(n, 10, \pm 3)-\Pi(n-1,10, \pm 3)]}{n^{s}} \\
& =\sum_{\substack{p \equiv \pm 1 \\
(\bmod 10)}} \frac{1}{p^{s}}-\sum_{\substack{p \equiv \pm 3 \\
(\bmod 10)}} \frac{1}{p^{s}}=\sum_{p} \frac{x(p)}{p^{s}} .
\end{aligned}
$$

By Lemma 1, the sum

$$
\sum_{n=2}^{\infty} P(n)\left(\frac{1}{n^{s}}-\frac{1}{(n+1)^{s}}-\frac{s}{n^{s+1}}\right)
$$

represents a regular function for $\sigma>0$. Since $P(I)=0$ it follows for $\sigma>1$, that 


$$
\begin{aligned}
\sum \frac{\chi(p)}{p^{s}} & =-\sum_{n=2}^{\infty} P(n)\left(\frac{1}{n^{s}}-\frac{1}{(n+1)^{s}}\right) \\
& =-s \sum_{n=2}^{\infty} \frac{P(n)}{n^{s+1}}+R_{9}(s), \\
\sum_{n=2}^{\infty} \frac{P(n)}{n^{s+1}} & =-\frac{1}{2 s} \log \left(s-\frac{1}{2}\right)+R_{10}(s) .
\end{aligned}
$$

Moreover, for $\sigma>\frac{1}{2}$ and $C$ a constant,

$$
\begin{aligned}
\sum_{n=2}^{\infty} \frac{(\sqrt{n} / \log n)}{n^{s+1}} & =\sum_{n=2}^{\infty} \frac{1}{(\log n) n^{s+\frac{1}{2}}} \\
& =\sum_{n=2}^{\infty} \frac{1}{\log n}\left(\frac{1}{n^{s+\frac{1}{2}}}-\frac{1}{n^{2+\frac{1}{2}}}\right)+C \\
& =\int_{s}^{2}\left(\zeta\left(n+\frac{1}{2}\right)-1\right) d u+C=\int_{s}^{2} \frac{d u}{u+\frac{1}{2}}+R_{11}(s) \\
& =-\log \left(s-\frac{1}{2}\right)+R_{12}(s) .
\end{aligned}
$$

Returning to the definition of $F(s)$, we have

$$
\begin{aligned}
F(s) & =\left(\frac{-1}{2 s}+1\right) \log \left(s-\frac{1}{2}\right)+R_{10}(s)-R_{12}(s) \\
& =\frac{1}{s}\left(s-\frac{1}{2}\right) \log \left(s-\frac{1}{2}\right)+R_{13}(s) .
\end{aligned}
$$

The last expression clearly displays the asserted properties of $F(s)$.

Q.E.D.

THEOREM 3. The following inequality holds for every $\delta>0$.

$$
\left|\frac{P(x)}{(\sqrt{x} / \log x)}-1\right|<\delta \text {; }
$$

that is, given any $\delta$ and $\xi>0$, there always exists an $x=x(\delta, \xi)>\xi$ such that

$$
-\delta \frac{\sqrt{x}}{\log x}<P(x)-\frac{\sqrt{x}}{\log x}<\delta \frac{\sqrt{x}}{\log x} .
$$

Proof (by contradiction). Suppose false and let

$$
Q(x)=\frac{P(x)}{(\sqrt{x} / \log x)}-1 \text {. }
$$


Then from a certain point on, $Q(x)$ will never belong to the interval $(-\delta, \delta)$. Considered as a function of increasing $x, Q(x)$ has the following properties:

(i) it is continuous on the open interval between two consecutive integers;

(ii) it is continuous on the right at every integer;

(iii) it either remains continuous or makes a jump equal to

$$
\lim _{\varepsilon \rightarrow 0}(Q(x)-Q(x-\varepsilon))= \pm \frac{1}{(\sqrt{x} / \log x)}
$$

when $x$ is an integer.

Thus,

$$
\lim _{x \rightarrow \infty}(Q(x)-Q(x-\varepsilon))=0,
$$

and we have two cases to consider, namely

$$
Q(x)>\delta \text { or } Q(x) \leq-\delta \text {. }
$$

Since the treatment of the two cases is quite similar we need only consider Case 1 in this paper. Thus, we let

$$
x \geq n_{0} \geq 2, n_{0} \text { an integer and } Q(x) \geq \delta \text {. }
$$

Then the Dirichlet series $F(s)$ of Theorem 2, which converges for $\sigma>1$, satisfies the hypothesis of Theorem $I$ for $\alpha=1$ and $B=\frac{1}{2}$. Thus $F(s)$ converges for $s>\frac{1}{2}$. Also, in the interval $\left[\frac{1}{2}, 1\right]$ we have

$$
\begin{aligned}
F(s)=\sum_{n=2}^{n} \frac{|P(n)-(\sqrt{n} / \log n)|}{n^{s+1}}+ & \sum_{n=n_{0}}^{\infty} \frac{\delta(\sqrt{n} / \log n)}{n^{s+1}} \\
& -\sum_{n=2}^{n_{0}^{-1}} \frac{|P(n)-(\sqrt{n} / \log n)|}{n^{\frac{3}{2}}}+\delta \sum_{n=n_{0}}^{\infty} \frac{1}{n^{s+\frac{1}{2}} \log n} .
\end{aligned}
$$

Since, $\sum_{n=2}^{\infty} I /(n \log n)$ diverges it follows that

$$
\lim _{8 \rightarrow \frac{1}{2}^{+}} F(8)=\infty
$$


But this contradicts Theorem 2 and hence proves Theorem 3 .

\section{References}

[1] P.L. Chebyshev, "Lettre de M. le professeur Tchébychev á M. Fuss, sur un noveau théorème relatif aux nombres premiers contenus dans les formes $4 n+1$ et $4 n+3 "$. a) Buzl. de la classe phys. math. de 2'Acad. Imp. des Sciences, St. Petersburg, 11 (1953), 208. b) Oeuvres, Tome I, 697-687. (Commissionaires Acad. Impér. Sci. St. Petersburg, 1899; reprint by Chelsea, New York, 1962).

[2] G.H. Hardy, A course in pure mathematics, 10th edition, (Cambridge University Press, Cambridge, 1952).

[3] S. Knapowskit and P. Turán, "Ü̉er einige Fragen der vergleichenden Primzahlentheorie", Abhandlungen cas Zahlentheorie und Analysis (zur Erinnerung an Edmund Landou (1877-1938)), herausgegeben von Paul Turán, (Deutsch. Verlag Wiss., Berlin, 1968).

[4] Edmund Landau, "Über einen Satz von Tschebyschef", Math. Ann. 61 (1905), 527-550.

[5] Edmund Landau, Handbuch der Lehre von der Vertei Zung der Primzahlen, 2 Bände. 2na ed. with an appendix by Paul T. Bateman. (Chelsea Publishing Company, New York, 1953).

[6] E. Phragmén, "Sur le logarithme intégral et la fonction $f(x)$ de Riemann", Öfversigt af Kongl. Vetenskaps - Akademiens Förhandlingar, Stockholm, 48 (1891), 599-616.

University of Kentucky, Lexington, Kentucky, USA. 